\title{
Study of Distributed Beamforming for Wireless Power Transfer in Presence of RF Impairments
}

\author{
Florin Huţu, Vincent Lechappé, Guillaume Villemaud, and Michaël Di Loreto
}

\begin{abstract}
For an equivalent performance (sensitivity, communication range, computational capability, etc.), the energy efficiency of connected devices is continuously increasing. Providing the energy for such objects by the means of electromagnetic waves (wireless power transfer) becomes a feasible option in various scenarios, especially those in which the use of conventional energy resources (e.g. batteries) is unfeasible. This paper tackles a particular method of wireless power transfer, which is the distributed beamforming, i. e. the use of several sources, spatially distributed and which are coordinating together in order to maximize the power of the electromagnetic field in a particular location. Through simulation results, this paper highlights the robustness of this approach to different nonidealities, i.e. frequency and phase synthesis, phase noise and channel characteristics. A first experimental setup is also presented.
\end{abstract}

\section{Introduction}

All studies predicted an exponential increase of the number of connected devices (50 billions in 2020 [1]) in a multitude of applications related to agriculture, industry, health monitoring, etc. This huge amount of connected devices gives an idea of how important is to find new ways to decrease their environmental impact.

In many Internet of Things (IoT) applications, the use of classical energy source is inappropriate. One can cite the case of sensors buried in concrete that are monitoring the mechanical efforts inside the walls. The sensor life span will be strictly related to the battery lifetime. For these scenarios, the solution to transmit the entire or a part of the energy through electromagnetic waves may be taken into account. Indeed, this solution is feasible because of the increasing efficiency of the electronic devices. Some studies (e.g. [2]) are predicting that in the next decade the energy needed for a computational task will decrease by a factor 10000 compared to the current levels.

Wireless power transfer has already been proposed as a method to energize connected objects. Generally, the research is focusing at the receiver side, in order to increase the efficiency of the RF power rectifier since here is the main limitation. This efficiency is varying with respect to the received input power. To the best of our knowledge, with the current technology, in order to have a rectifier's efficiency greater than $25 \%$, the received signal should be superior to $-20 \mathrm{dBm}$ [3].

In order to increase the rectifier's efficiency, some recent works are focusing on the use of multi-carrier signals [4]. Indeed, such signals are renowned for having a high PAPR (Peak to Average Power Ratio) and to increase the DC voltage level at the rectifier's output [5]. Some other works are proposing a systemic view on maximizing the efficiency of the WPT by taking into account the power source, the effects of the propagation channel and the characteristics of the rectifier [6].

Wireless power transfer by using time reversal technology was proposed in [7]. This technique is based on spatial-temporal focusing effect. After a channel probing, made by sending an impulse signal, the transceiver will return a time-reversed, conjugate and amplified replica of the received impulse. These replicas will combine at the exact spot of the initial pulse, bringing there a relatively high amount of electromagnetic field energy.

Distributed beamforming has already been proposed as a way to maximize the wireless power transfer and some theoretical solutions are proposed [8]. Here, the authors are formulating the problem of maximizing the power delivered by a distributed antenna array to a receiver by taking into account the channel effects. A fixed-point algorithm is giving the optimum power allocations in a finite set of frequencies.

In distributed beamforming, a network of individual wireless nodes having single antennas are cooperating in order to generate synchronized signals. The considered sources may be Access Points (AP) in Wireless Local Area Networks (WLAN) or cellular base stations. The initial phase of the emitted signals are chosen such way that, in a desired spot, the phase coherence occurs. Consequently, the amplitude of the electromagnetic field is locally maximized. The main contribution of this paper is to study the impact of the RF impairments on the amount of energy transferred by employing distributed beamforming. More precisely, by taking as reference an ideal scenario, the power decrease in presence of frequency and phase synthesis errors, in presence of sources phase noise and in presence of a realistic propagation channel is studied.

In Section 2, the distributed beamforming for wireless power transfer purpose is formalized. Then, some simulation results tackle the impact of different beam- 
forming synthesis errors on the received power level. Section 3 presents an experimental setup built in order to validate the distributed beamforming scenarios. Conclusion and future directions are given in Section 4.

\section{Problem statement and simulation results}

Consider $N$ identical sources randomly distributed in a Cartesian plane. These sources are coordinating together in order to obtain phase coherent signals at the origin of the coordinate plane in order to perform wireless power transfer efficiently. Each source is defined by its coordinates $\left(x_{i}, y_{i}\right)$ where $i \in[1, N]$. Consider also that each source is able to generate a signal at a frequency $f_{0}$ and with an identical power level $P$. At the center of the coordinate system, a receiver having low energy resources is placed.

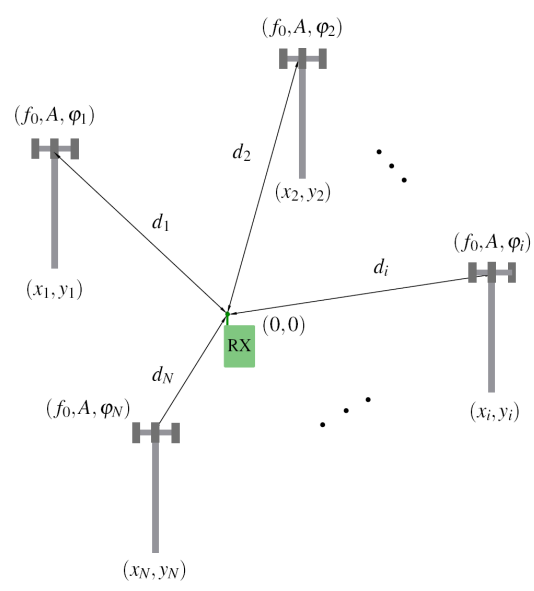

Figure 1: Scenario of wireless power transfer using geographically distributes RF sources

This receiver is power supplied by the electromagnetic field produced by the $N$ sources. As stated, the power of the received electromagnetic field is maximized if the arriving signals are in phase coherence. In order to obtain the phase coherence at the receiver level, the initial phase of the emitted signals should be :

$$
\varphi_{i}=\frac{2 \pi}{\lambda_{0}} \sqrt{x_{i}^{2}+y_{i}^{2}}
$$

where $\lambda_{0}$ is the wavelength of the emitted waveform.

In the case of the phase coherence, the power level collected by the receiver increases with the number of sources. In the particular case in which $N$ sources are placed at the same distance around the receiver, the gain related to the power level received from one source can be expressed as:

$$
[G]_{d B}=20 \cdot \log _{10}(N) .
$$

Indeed, suppose that only one source is emitting. The received power level can be written as:

$$
\left[P_{R}\right]_{d B m}=10 \cdot \log _{10}\left(\frac{A^{2}}{R}\right)
$$

where $A$ is the amplitude of the received sinusoidal waveform and $R$ is the load presented by the receiver.

Consider now that all $N$ sources are emitting sinusoidal waveforms at the same frequency so that the signals arrive in phase coherence at the receiver level. In this case, the received power level become:

$$
\left[P_{R N}\right]_{d B m}=10 \cdot \log _{10}\left[\frac{(N \cdot A)^{2}}{R}\right] .
$$

If the gain in terms of power $G$ is defined as :

$$
G \triangleq \frac{P_{R N}}{P_{R}}
$$

then, the result given in equation (2) is straightforward. As an example, from Figure 2, which is the graphical representation of equation (2), one can remark that when the four sources are emitting, the gain in terms of received power is $12 \mathrm{~dB}$.

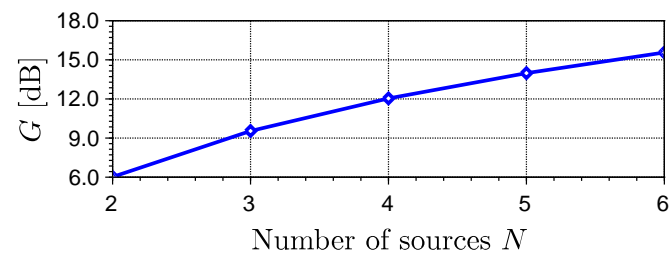

Figure 2: The gain in terms of received power

In order to verify this theoretical gain in a more realistic scenario, a simulator based on Keysight's ADS software was implemented. In this simulator, each source is placed at a certain distance from the sink and is able to deliver a CW (continuous wave) signal. Between the different sources and the sink, free space path loss propagation channel was considered as a first step.

If the scenario given in Table 1 is simulated, the received signals have an identical phase of 150.8 degrees and the received power level is $-33.04 \mathrm{dBm}$. In this particular case, the emitted power levels are of $-20 \mathrm{dBm}$ and antenna gains are $4.1 \mathrm{dBi}$. Cables connecting different antennas and having an insertion loss of $0.85 \mathrm{~dB}$ were also taken into account.

Moreover, the simulator is able to test the impact on the received power of the frequency and phase synthesis deviation. Figure 3 presents the variation of the received power level if one of the emitters among the four has a 


\begin{tabular}{|c|c|c|c|}
\hline Antenna & x (meters) & y (meters) & $\varphi$ (degrees) \\
\hline \hline 1 & -0.75 & 0.6 & 0 \\
\hline 2 & 0.75 & 0.7 & 68.2 \\
\hline 3 & -0.65 & -0.75 & 33.4 \\
\hline 4 & 0.85 & -0.85 & -108.2 \\
\hline
\end{tabular}

Table 1: Coordinates and the initial phases of the CW sources

frequency deviation $\Delta_{f}$ with respect to the central frequency $f_{0}=868 \mathrm{MHz}$. As can be remarked, compared to the maximum power level $(-33 \mathrm{dBm})$, the received power level is about $1 \mathrm{~dB}$ lower for deviations of the emitted frequency around $\pm 25 \mathrm{kHz}$ with respect to the ideal value. This value corresponds to a variation of the synthesized frequency of approximately $28.8 \mathrm{ppm}$ (parts per million) and shows the sensitivity of the distributed beamforming strategy to frequency variations.

Figure 4 presents the impact of the phase deviation on the received power level. More precisely, the phase of one emitter is modified with respect to the desired value, while the other three phases are remaining at their ideal values. The received power level is insensitive on the phase synthesis since power diminutions of $1 \mathrm{~dB}$ are obtained for errors $\Delta \varphi$ greater than \pm 60 degrees, with respect to the ideal phase $\varphi_{i}$.

These simulation results are showing the consequence of the synchronization between the different sources which seems to be more important than the one of phase synthesis.

Another parameter which was suspected to have an influence on the distributed beamforming accuracy was the local oscillator's phase noise. Indeed, real oscillators are presenting random variations of their instantaneous

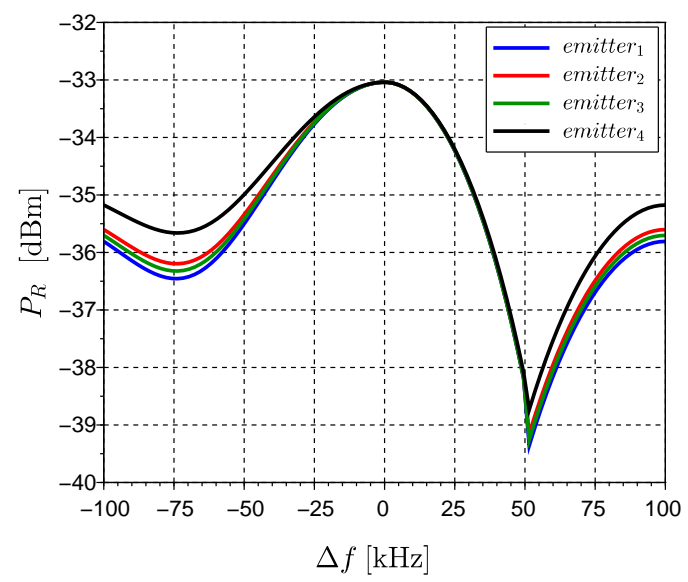

Figure 3: The received power level variation with respect to the CFO (Central Frequency Offset)

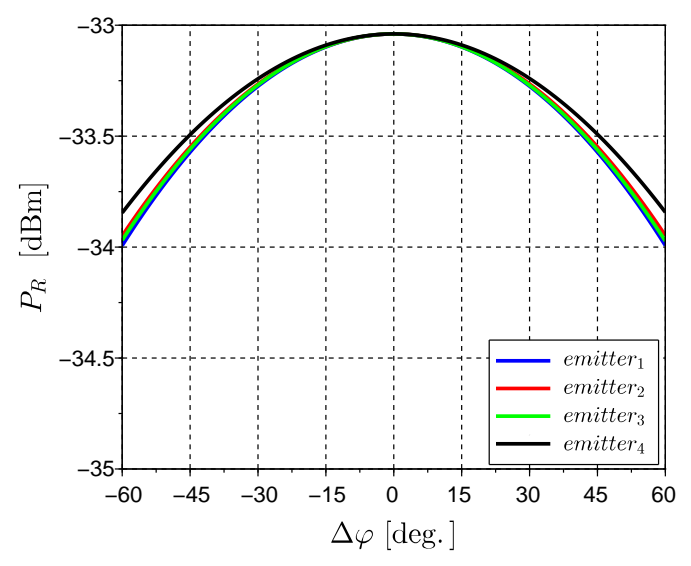

Figure 4: The received power level variation with respect to the deviation of the desired phases $\varphi_{i}$

phase which may corrupt the phase accuracy at the receiver side. In order to test this influence, the phase noise of the NI PXIe-5644R RF vector signal transceiver was considered as reference. This transceiver has good performance in terms of phase noise and it is expected that the different sources used for distributed beamforming will present poor performance. The VST's phase noise levels are given in Table 2.

\begin{tabular}{|c||c|c|c|c|c|}
\hline Freq. [kHz] & $10^{-1}$ & $10^{0}$ & $10^{1}$ & $10^{2}$ & $10^{3}$ \\
\hline \hline PN [dBc/Hz] & -83 & -101 & -104 & -115 & -143 \\
\hline
\end{tabular}

Table 2: NI's 5644 VST's phase noise

Figure 5 presents the variation of the received power level when the phase noise floor is increased with respect to the values given in Table 3. A parametric simulation was performed while increasing the power level of the phase noise with a quantity $P_{N}$. All the four sources are considered to have identical phase noise levels. As can be remarked, a phase noise of $-38 \mathrm{dBc} / \mathrm{Hz} @ 100 \mathrm{~Hz}$ will decrease the received power level by $1 \mathrm{~dB}$ compared to the ideal value. One can say that the local oscillator's

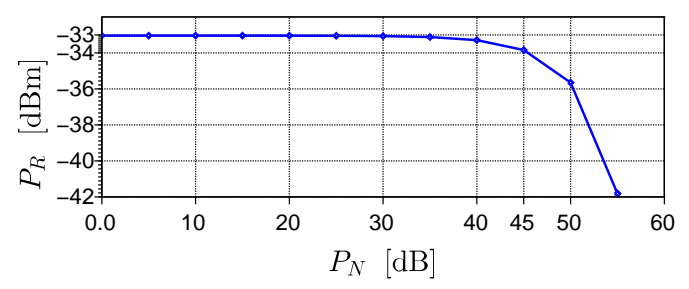

Figure 5: The received power variation with respect to the increase of the phase noise level 
phase noise has a small impact on the accuracy of the distributed beamforming performance since this value is beyond the ones announced for most of the commercial radio front-ends. Moreover, this result reinforces the remark that distributed beamforming is robust in terms of phase synthesis.

The propagation channel plays also an important role in the accuracy of the phase coherence at the receiver side. In a realistic distributed beamforming scenario, multipath phenomena between the emitters and the receiver are unavoidable. Propagation channels having multiple paths (Rice channels) were considered between each emitter and the receiver.

The Rician factor $\alpha \in[0 \cdots 1]$ was considered as in [9], being the ratio between the amplitude of the LOS (line of sight) power level and the power of the NLOS (non line of sight) version. Moreover, the NLOS path was considered to be delayed by a factor $\tau \in[0 \cdots T]$, where $T=1 / f_{0}$ is the period of the emitted signals.

As can be remarked from Figure 6, a multi path channel is decreasing the received power level only if the NLOS version of the emitted signal is closed to quadrature (i. e. $\tau \approx T / 2$ ). This degradation is more important when the rician factor $\alpha$ is high (i. e. power level of the NLOS received signal closed to the LOS power level).

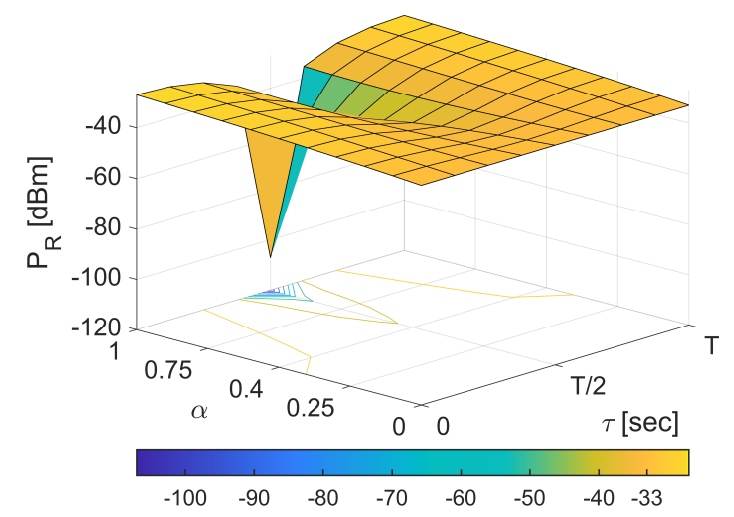

Figure 6: Influence of the propagation channel on the received power level

\section{Experimental setup}

A distributed beamforming scenario was implemented in FIT/CorteXlab testbed [10], a quasi-anechoic environment. A National Instruments' PXI chassis containing four $5645 \mathrm{VST}$ s is the core of the experimental setup. The four VST (Vector Signal Transceiver) were used as CW generators and a spectrum analyzer was employed in order to measure the received power level.

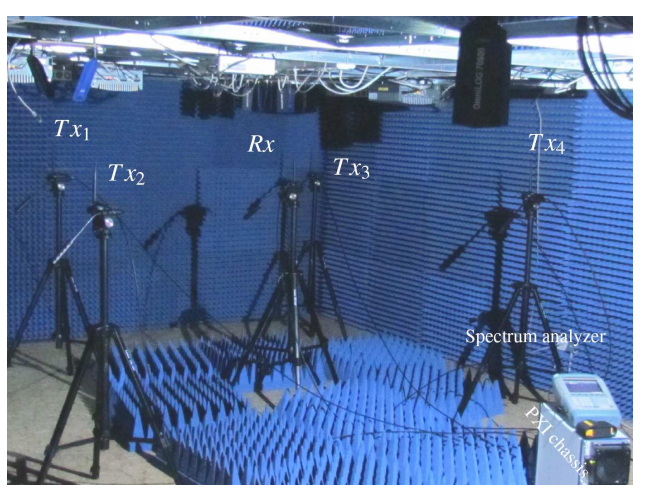

Figure 7: Measurement setup deployed in an quasianechoic chamber

Experiments were conducted at $868 \mathrm{MHz}$ and, as can be seen from Figure 7, four omnidirectional antennas have been connected to the VST's RF output. The antennas have a gain of $4.1 \mathrm{dBi}$, the coaxial cables have the same phase shift at $868 \mathrm{MHz}$ and the emitted power level was set to $-20 \mathrm{dBm}$, identical for the four VST. One receiving omnidirectional antenna has been placed at the center of a Cartesian coordinate system. The synchronization of the four VST and their phase coherence were tested beforehand.

More precisely, the phase coherence was assured by sharing the local oscillator of one of the VSTs to the other three. A calibration procedure was implemented in order to cancel phase offset brought by the coaxial cables connecting the VTS's local oscillators inputs and outputs.

The scenario presented in Table 1 was implemented and the received power level was approximately -34.5 $\mathrm{dBm}$, close to the simulated one. The difference between the simulated and measured received powers may come from the antenna gains uncertainty.

\section{Conclusion}

This article presented the distributed beamforming as a solution for wireless power transfer. The impact of the RF chain non idealities on the received power level is also tackled. Moreover, the paper presented an experimental setup able to implement distributed beamforming scenarios. Future work will focus on implementation of algorithms able to take into account the impact of the propagation channel since it is the most unpredictable part of the distributed beamforming setup.

\section{Acknowledgment}

This work was supported by the "Institut National des Sciences Appliquées" Lyon, France under the BQR 2019-2021 program. 


\section{References}

1. "More Data, Less Energy, Making Network Standby More Efficient in Billions of Connected Devices," International Energy Agency, 2014.

2. J. Koomey, S. Berard, M. Sanchez, and H. Wong, "Implications of Historical Trends in the Electrical Efficiency of Computing," IEEE Annals of the History of Computing, vol. 33, no. 3, pp. 46-54, March 2011.

3. Humberto P. Paz and Vinicius S. Silva and Eduardo V.V. Cambero and Humberto X. Araújo and Ivan R.S. Casella and Carlos E. Capovilla, "A survey on low power RF rectifiers efficiency for low cost energy harvesting applications," AEU - International Journal of Electronics and Communications, vol. 112, p. 152963, 2019.

4. R. Rousseau, F. Hutu, and G. Villemaud, "On the use of Vector Fitting and State-Space Modeling to Maximize the DC Power Collected by a Wireless Power Transfer System," in 2018 2nd URSI Atlantic Radio Science Meeting (AT-RASC), May 2018, pp. 1-4.

5. A. Boaventura, D. Belo, R. Fernandes, A. Collado, A. Georgiadis, and N. B. Carvalho, "Boosting the Efficiency: Unconventional Waveform Design for Efficient Wireless Power Transfer," IEEE Microwave Magazine, vol. 16, no. 3, pp. 87-96, April 2015.

6. B. Clerckx, R. Zhang, R. Schober, D. W. K. Ng, D. I. Kim, and H. V. Poor, "Fundamentals of Wireless Information and Power Transfer: From RF Energy Harvester Models to Signal and System Designs," IEEE Journal on Selected Areas in Communications, vol. 37, no. 1, pp. 4-33, Jan 2019.

7. M. Ku, Y. Han, H. Lai, Y. Chen, and K. J. R. Liu, "Power Waveforming: Wireless Power Transfer Beyond Time Reversal," IEEE Transactions on Signal Processing, vol. 64, no. 22, pp. 5819-5834, Nov 2016.

8. S. Goguri, D. Ogbe, R. Mudumbai, D. Love, S. Dasgupta, and P. Bidigare, "Maximizing wireless power transfer using distributed beamforming," in 2016 50th Asilomar Conference on Signals, Systems and Computers, Nov 2016, pp. 1775-1779.

9. A. Abdi, C. Tepedelenlioglu, M. Kaveh, and G. Giannakis, "On the estimation of the K parameter for the Rice fading distribution," IEEE Communications Letters, vol. 5, no. 3, pp. 92-94, 2001

10. FIT/CorteXLab website. [Online]. Available: http://www.cortexlab.fr

First and Third Authors are with Univ Lyon, INSA Lyon, Inria, CITI, F-69621, Villeurbanne, France; e-mails: florindoru.hutu@insa-lyon.fr; guillaume.villemaud@insa-lyon.fr.

Second and Fourth Authors are with Université de Lyon, Université Claude Bernard Lyon1, CNRS, Ampère, F-69622, Villeurbanne, France; e-mail: vincent.lechappe@insa-lyon.fr; michael.di-loreto@insa-lyon.fr. 